\title{
Huntington's disease treatment with memantine prevented the progression of chorea movement
}

\author{
Kazumasa Saigoh ${ }^{1}$, Makito Hirano ${ }^{2}$, Yoshiuki Mitsui ${ }^{2}$, Ikegawa Atsuko ${ }^{1}$, Itsuki Oda ${ }^{1}$, \\ Makoto Samukawa $^{2}$, Keisuke Yoshikawa ${ }^{2}$, Yuko Yamagishi ${ }^{2}$, Susumu Kusunoki², and \\ Yoshitaka nagai ${ }^{2}$ \\ ${ }^{1}$ Kindai University Hospital \\ ${ }^{2}$ Kindai University Faculty of Medicine Graduate School of Medical Sciences
}

May 6, 2021

\begin{abstract}
We have recorded the Huntington's disease patient's gait videos at the 5 years. It's treatment with memantine prevented the progression of Clinical symptom. It is recognized to be improved blood flow image in front-temporal lobe of IMP-SPECT.

Huntington's disease treatment with memantine prevented the progression of chorea movement

Kazumasa Saigoh ${ }^{1,2 *}$, Makito Hirano ${ }^{1}$, Yoshiyuki Mitsui ${ }^{1}$, Atsuko Ikegawa ${ }^{2}$, Itsuki Oda ${ }^{2}$, Makoto Samukawa ${ }^{1}$, Keisuke Yoshikawa ${ }^{1}$, Yuko Yamagishi ${ }^{1}$, Susumu Kusunoki ${ }^{1}$, Yoshitaka Nagai ${ }^{1}$

1) Department of Neurology, Faculty of Medicine, Kindai University, Japan.

2) Department of Clinical genetics, Kindai University Hospital, Japan.

*Corresponding Author

Kazumasa Saigoh

Department of Neurology, Faculty of Medicine, Kindai University, Japan.

377-2, Ohno-Higashi, Osaka-Sayama, Osaka 589-8511, Japan

E-mail: saigohk@yahoo.co.jp saigohk@gmail.com

Funding information

This research received "The Research Foundation for Dementia of Osaka (2020-4)" grant from a public funding agency.

Abstract

We have recorded the Huntington's disease patient's gait videos at the5 years. It's treatment with memantine prevented the progression of Clinical symptom. It is recognized to be improved blood flow image in fronttemporal lobe of IMP-SPECT.Keywords: Huntington's disease, Memantine, SPECT, Alzheimer's disease,

Videos record

Introduction
\end{abstract}


Huntington's disease is characterized by personality changes, such as irritability and restlessness, and mental symptoms, such as hallucinations and delusions. When the personality changes become noticeable, involuntary movements called chorea develop. ${ }^{1}$ The disease is inherited through autosomal dominance and is caused by the expansion of the CAG repeat sequence inHTT gene coding region. The diagnosis is based on the presence of this extension. The average age at onset of Huntington's disease is 35 to 44 years. In approximately two-thirds of affected patients, neurological symptoms first develop, but in others, the first symptoms are psychological changes. In the early stage after diagnosis, slight changes appear in eye movements, chorea movements, as do inconspicuous involuntary movements, positioning of the head, depressive symptoms, and irritability. ${ }^{2}$ Chorea movements can be partially suppressed by haloperidol and tetrabenazine of the monoamine-depleting agent. ${ }^{1}$

In our case, Huntington's disease treated with memantine, who did not show rapid progression of neurological symptoms, such as chorea movement and gait balance for 5 years. Furthermore, IMP-SPECT revealed improved blood flow image in the front-temporal lobe by memantine treatment.

\section{Case presentation}

The patient was a 75-year-old woman (II-6). On her first visit to our hospital at the age of 75 years, Huntington's disease was suspected because she exhibited chorea in the neck and upper limbs and her family history included Huntington's disease. Therefore, with the patient's consent, genetic testing was performed; the results revealed a repeat abnormality of the HTT gene; thus, she was diagnosed as Huntington's disease. The psychological test results were as follows: 25 points on the Mini-Mental State Examination and 10 points on the Alzheimer's Disease Assessment Scale-Cognitive Subscale. Tetrabenazine, the only drug capable of preventing chorea movement, was then administered, but nausea and fatigue necessitated the discontinuation of oral administration. The patient's older brother (II-5) who developed the disease at the same age as her had chorea that rapidly progressed; 5 years later, he was almost immobile and bedridden (Fig1).

Magnetic resonance imaging of the head showed changes in the striatum. In a cerebral blood flow test (IMPSPECT), decreased blood flow was observed in the precuneus (anterior wedge) and posterior cingulate gyrus (Fig. 2). Usually, such areas of decreased blood flow are observed in Alzheimer-type dementia patients. We suspected Alzheimer-type dementia complications based on SPECT results of patient.

Initially, donepezil administration was considered, but because the patient was irritable, a regimen of lowdose memantine ( $5 \mathrm{mg} /$ day) was started. Thereafter, swallowing and oral symptoms improved. Five years since then, clinical symptoms have not worsened. Involuntary movements also have not worsened substantially, and we have recorded the patient's gait videos at the age of 75 and 80 years (Video1A and 1B). The patient did not show progression in gait speed and balance. Also after 5years, IMP-SPECT revealed improved blood flow image in front-temporal lobe. Additionally, information provided by family members and medical examinations by physicians specializing in rehabilitation did not reveal deterioration in the swallowing function or a decrease in the activities of daily living.

\section{Discussion and conclusions}

We conducted cerebral blood flow tests and cognitive function tests over 5 years in the patient to document the disease's slow progression. The cognitive and behavioral symptoms exhibited by patients with Huntington's disease are more similar to those exhibited by patients with frontotemporal dementia than those exhibited by patients with Alzheimer's disease. ${ }^{3}$ In our patient, therefore, cerebral blood flow examination indicated Alzheimer's-type dementia. In general, many patients with Huntington's disease show cognitive decline. Over time, involuntary movements increase, and communication ability is affected by deterioration in voluntary cognitive writing ability and speech. Thus, the patient may understand the content of evaluator's questions, but involuntary movements causing dysarthria may impair expression and communication. ${ }^{3}$ Therefore, we believe that in patients with Huntington's disease at old advanced age, IMP-SPECT should be performed. Because elderly people may exhibit different types of dementia such as Alzheimer's-type dementia, Lewy body dementia, and frontotemporal dementia. IMP-SPECT revealed that our elderly female patient with Huntington's disease also had Alzheimer's-type dementia. 
Recently in Japan, tetrabenazine reportedly improved Huntington's disease-associated chorea; however, symptoms other than chorea did not improve. Tetrabenazine depletes monoamines at nerve endings (dopamine, serotonin, and noradrenaline) by selectively inhibiting monoamine vesicle transporter 2 at presynaptic receptors of the central nervous system. ${ }^{4}$ The effect of tetrabenazine on chorea results mainly from dopamine depletion in the striatum, which is the main lesion site of Huntington's disease. In our patient, however, oral administration caused nausea and fatigue, and the drug had to be discontinued. So considering her dementia symptoms and IMP-SPCT results, we prescribed $5 \mathrm{mg}$ memantine because it has shown effects in Alzheimer's-type dementia.

Memantine is an $N$-methyl-d-aspartate (NMDA) receptor inhibitor that is usually administered to improve cognitive function in Alzheimer's-type dementia ${ }^{5}$. Recently, its additional effects such as improving swallowing function, lip dyskinesia, and chorea-like symptoms have been reported. ${ }^{5}$ Several patients with Huntington's disease were administered $20 \mathrm{mg}$ memantine and ameliorating effects were observed. ${ }^{6}$ Moreover, two memantine clinical trials are ongoing for Huntington's disease. ${ }^{7}$ Furthermore, it has been used for treating other neurodegenerative diseases. The NMDA receptor antagonist amantadine has long been used not only as an anti-parkinsonism drug but also for treating dyskinesia.

Tetrabenazines, which are involved in the regulation of monoamine receptors, have recently been approved by the US Food and Drug Administration for treating tardive dyskinesia ${ }^{8}$ and implicate a possible mechanism for chorea in Huntington's disease. Similarly, the administration of memantine, an NMDA receptor inhibitor, may alleviate motor symptoms because monoamine and NMDA receptor regulation is closely related in the brain.

In our patient, dysphagia and dysarthria were not exacerbated for 5 years after the oral administration of $5 \mathrm{mg}$ memantine. Most memantine clinical trials focusing on Huntington's disease have been evaluations of cognitive improvement; no detailed observational study of motor symptoms has been conducted. ${ }^{7}$ The patient's older brother (II-5 in Fig. 1) developed the disease at about the same time as her, but the disease progressed rapidly in him over 5 years; he frequently had aspiration pneumonia and was unable to walk. Activities of daily living were almost completely assisted. Conversely, our patient (II-8 in Fig. 1) remained ambulatory, and she could maintain activities of daily living to the extent that she could take a bath and use the toilet without assistance.

Memantine has been reported to induce symptoms such as myoclonus ${ }^{9}$; conversely, it is effective against other motor symptoms and dystonia. One of the sites targeted by memantine is thought to be the dysfunctional glutamatergic neurotransmitter system via the NMDA receptor. ${ }^{10}$ In our patient, a small amount of memantine may have been effective in slowing Huntington's disease progression.

Memantine was reportedly effective in a small number of Huntington's disease patients (Cankurtaran et al., 2006); in patients with the $H T T$ gene who have not yet developed symptoms, memantine may suppress symptom worsening in the future (Leavitt, 2011). Findings in our patient suggest that oral memantine has some influence on Huntington's disease motor symptoms.

We recorded changes over time in 5 years, such as to be improved blood flow image in in front-temporal lobe of IMP-SPECT. It's treatment with memantine prevented the progression of Clinical symptom in 5years period of videos. We believe that this report will provide clinicians with valuable information in diagnosing and treating Huntington's disease.

\section{Author Contributions:}

KS conceived the idea of the study and its design. AI, IO, KY, YY and KS collected the Clinical data. KS drafted the manuscript. MH, YM, MS, SK, YN reviewed edited the manuscript for intellectual content. All authors approved the final manuscript.

\section{Conflicts of interest}

Not applicable 


\section{Ethics approval and consent to participate:}

This study was conducted with the approval of the Ethics Committee of Kindai University, Osaka, Japan (number 16-011).

\section{Consent for publication}

Written informed consent was obtained from the patient for publication. A copy of the written consent is possible for review by the editor.

\section{Reference}

1. Ross, C. A., E. H. Aylward, E. J. Wild, D. R. Langbehn, J. D. Long, J. H. Warner, et al. 2014. Huntington disease: Natural history, biomarkers and prospects for therapeutics. Nat. Rev. Neurol. 10: 204-216.

2. Liu, D., J. D. Long, Y. Zhang, L. A. Raymond, K. Marder, A.Rosser, et al. 2015. Motor onset and diagnosis in Huntington disease using the diagnostic confidence level. J. Neurol. 262: 2691-2698.

3. Bates, G. P., R. Dorsey, J. F. Gusella, M. R. Hayden, C. Kay, B. R. Leavitt, et al. 2015. Huntington disease. Nat. Rev. Dis Primers, 1:15005.

4. Ross, C. A., and S. J. Tabrizi, 2011. Huntington's disease: From molecular pathogenesis to clinical treatment. Lancet. Neurol. 10: 83-98.

5. Johanssen, T., N. Suphantarida, P. S. Donnelly, X. M. Liu, S. Petrou, A. F. Hill, et al. 2015. PBT2 inhibits glutamate-induced excitotoxicity in neurons through metal-mediated preconditioning. Neurobiol. Dis. 81: $176-185$.

6. Cankurtaran, E. S., E. Ozalp, H. Soygur, and A. Cakir, 2006. Clinical experience with risperidone and memantine in the treatment of Huntington's disease. J. Natl. Med. Assoc. 98: 1353-1355.

7. Leavitt, B., and Huntington Study Group. 2011. A trial of memantine as symptomatic treatment for early Huntington disease (MITIGATE-HD). Clinical. Trials. gov. database. Identifier. NCT01458470.

8. Aguilar, L., C. Lorenzo, R. Fernández-Ovejero, C. Roncero, and A. L. Montejo, 2019. Tardive dyskinesia after aripiprazole treatment that improved with tetrabenazine, clozapine, and botulinum toxin. Front in Pharmacol. 10: 281.

9. Kitagawa, N., and A. Takeuchi, 2014. Memantine-induced myoclonus. Neurology. 83: 1387.

10. Milnerwood, A. J., C. M. Gladding, M. A. Pouladi, A. M. Kaufman, R. M. Hines, J. D. Boyd, et al. 2010. Early increase in extrasynaptic NMDA receptor signaling and expression contributes to phenotype onset in Huntington's disease mice. Neuron. 65: 178-190.

\section{Figure Legends}

Figure 1. The patient's family tree.

Figure 2. (a) N-isopropyl-p-iodoamphetamine-single-photon emission computed tomography (IMPSPECT) three-dimensional stereotactic surface projection (3D-SSP) showing a decrease in cerebral blood flow on the date of diagnosis (at the age of 75 years). (b) Follow-up data (at the age of 80 years). Changes in cerebral blood flow are indicated in red and yellow. White arrow indicates precuneus and posterior cingulate gyrus.

Video 1. (a) Chorea Symptom Video on the date of diagnosis (at the age of 75 years). (b) Chorea Symptom Video at the follow-up date (at the age of 80 years). 


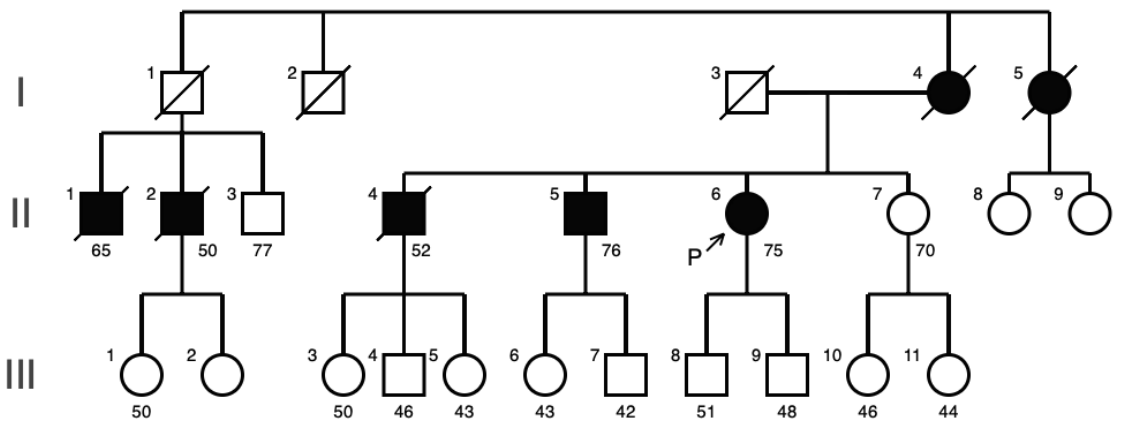

\section{Hosted file}

Fig2 a.b.pdf available at https://authorea.com/users/412272/articles/521017-huntington-sdisease-treatment-with-memantine-prevented-the-progression-of-chorea-movement

\section{Hosted file}

Huntingtons disease treatment_video1a.mp4 available at https://authorea.com/users/412272/ articles/521017-huntington-s-disease-treatment-with-memantine-prevented-the-progressionof-chorea-movement

\section{Hosted file}

Huntingtons disease treatment_video1b.mp4 available at https://authorea.com/users/412272/ articles/521017-huntington-s-disease-treatment-with-memantine-prevented-the-progressionof-chorea-movement 